\title{
QCD with zero, two and four flavors of light quarks - results from QCDSP
}

\author{
Chengzhong Sui ${ }^{\mathrm{a} *}$

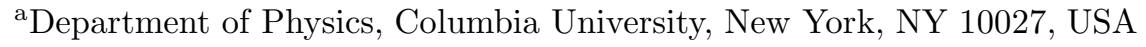

We present the results from full QCD simulations with four flavors of light staggered dynamical quarks on QCDSP supercomputer. Previous results are reproduced and the simulation reported here yields new results consistent with our previous runs. The hadron spectrum obtained with Wilson valence fermions reported here will allow us to determine if our earlier conclusions are independent of lattice formalism.

\section{INTRODUCTION}

It is well known that the number of light quark flavors $N_{f}$ governs the order of the QCD phase transition at finite temperature. On the other hand the effects of dynamical quarks at zero temperature have generally been found to be quite small when zero-flavor (quenched) and two-flavor QCD simulations are compared. One important question is whether this will continue as $N_{f}$ increases. In the past few years the Columbia group has done extensive comparison studies of zeroflavor, two-flavor and four-flavor QCD at zero temperature with the lattice spacing and volume fixed in physical units determined by the $\rho$ mass. What we found was that for four flavors the hadron spectrum and chiral condensate were significantly different from zero and two flavors: the hadronic spectrum appeared to be parity doubled and the chiral condensate was almost four times smaller than that for two flavors [1] [2].

Those calculations were done on the Columbia 16 Gflop machine, Fermi256. With the new generation of Columbia supercomputer, $Q C D S P$, we have been able to continue our flavor-dependence study. As a first step we need to check the reliability of our new hardware and software. We have reproduced results from several other groups [3] on

\footnotetext{
*This work was done in collaboration with Igor Arsenin, Dong Chen, Ping Chen, Norman H. Christ, Robert Edwards, George Fleming, Alan Gara, Sten Hansen, Chulwoo Jung, Adrian Kahler, Anthony Kennedy, Gregory Kilcup, Yubing Luo, Catalin Malureanu, Robert D. Mawhinney, John Parsons, James Sexton, Pavlos Vranas and Yuri Zhestkov. This work was supported in part by the Department of Energy. Presented on Lattice '98.
}

small lattices, and we have also tried to reproduce our own earlier results. For our previous fourflavor calculations we did two simulations with dynamical quark mass 0.01 and 0.02 . It is natural to have at least one more data point to be able to do a linear fit to the dynamical results. Another interesting question is whether the partial chiral symmetry restoration that we observed is just an artifact of the lattice formalism. To investigate this problem we have also measured the Wilson valence spectrum on these lattices generated with staggered dynamical quarks.

\section{SIMULATIONS AND RESULTS}

Table 1 lists the run parameters for our fourflavor simulations. Run I and III are our previous simulations done on Fermi256 [4] while II and IV are simulations performed on $Q C D S P$. Run IV is a check of results from $Q C D S P$ against previous results from Fermi256. Run II is a new simulation, adding one more dynamical data point so that linear fits can be performed. The four-flavor simulations used an exact hybrid Monte Carlo algorithm, employing the $\Phi$ hybrid molecular dynamics algorithm of [5], with a Monte Carlo accept/reject step.

Comparing the hadron masses in run III and IV in Table 1, we find that the hadron spectrum agrees very well statistically between the two machines. The expectation values of average plaquette and chiral condensate are also found to be consistent. However there is one discrepancy between the two calculations which we don't understand. In order to achieve comparable accep- 
Table 1

Simulation parameters for runs with $N_{f}=4$ and $\beta=5.4$ on $16^{3} \times 32$ lattice and corresponding results of staggered hadron masses with $m_{\mathrm{val}}=m_{\mathrm{dyn}}$. We have chosen the hadron mass fitting range typically from 9 to 16 for the $\pi$ and from 6 to 16 for the other particles.

\begin{tabular}{|c|c|c|c|c|}
\hline \multicolumn{5}{|c|}{$N_{f}=4, \beta=5.4$} \\
\hline run & I & II & III & IV \\
\hline$m_{\text {dyn }} a$ & 0.01 & 0.015 & 0.02 & 0.02 \\
\hline run length & 4450 & 4390 & 2725 & 3980 \\
\hline step size & 0.0078125 & 0.005 & 0.01 & 0.005 \\
\hline steps per trajectory & 64 & 100 & 50 & 100 \\
\hline acceptance & 0.95 & 0.87 & 0.99 & 0.91 \\
\hline machine & Fermi256 & $Q C D S P$ & Fermi256 & $Q C D S P$ \\
\hline \hline$\pi$ & $0.292(5)$ & $0.320(4)$ & $0.357(3)$ & $0.356(2)$ \\
\hline$\rho$ & $0.438(8)$ & $0.470(5)$ & $0.499(6)$ & $0.501(4)$ \\
\hline$a_{1}$ & $0.493(7)$ & $0.562(10)$ & $0.617(14)$ & $0.641(9)$ \\
\hline$N$ & $0.690(21)$ & $0.742(9)$ & $0.773(10)$ & $0.781(5)$ \\
\hline$N^{\prime}$ & $0.731(16)$ & $0.802(15)$ & $0.872(13)$ & $0.912(16)$ \\
\hline
\end{tabular}

tance rate with the new code we had to use a HMC time step size that was nearly half as large as that used previously. This is most likely due to some failure in our earlier program to adhere to the conventions of [5]. We are trying to resolve this discrepancy.

In figure 11 we plot the staggered hadron masses of $\rho$ and nucleon and their parity partners as a function of the dynamical quark mass using results from simulation I, II and IV. To confirm the near degeneracy of the parity partners in the zero quark mass limit we have done a 3-parameter fit to the 6 data points for both parity partner pairs, forcing a common intercept in the chiral limit respectively. The fits have $\chi^{2} / d o f=1.2$ for the $\mathrm{N}$ and $\mathrm{N}^{\prime}$ data and $\chi^{2} / \operatorname{dof}=0.7$ for the $\rho$ and $a_{1}$, which strongly support our parity doubling observation.

The degeneracy of parity partners implies a restoration of chiral symmetry breaking. Figure 2 shows $\langle\bar{\psi} \psi\rangle$ as a function of the dynamical quark mass for both $N_{f}=4$ and $N_{f}=2$, where the four-flavor data (solid circle) is taken from run I, II and IV and the two-flavor data (open squares) from [2]. A linear fit to the chiral condensate for four flavors (solid line) yields $\langle\bar{\psi} \psi\rangle=0.00278(33)$ in the zero-mass limit, which is more than three times smaller than $\langle\bar{\psi} \psi\rangle=0.00854$ (17) for two flavors. This indicates significant weakening of chiral symmetry breaking as the number of flavors increases, although the four-flavor value is still about eight standard deviations from zero. The non-vanishing chiral condensate in the zeromass limit suggests that the hadron mass splittings between the parity partners will not be exactly zero although they are invisible in figure 1 because of the relatively large statistical errors on the hadron masses.

In calculating the Wilson valence hadron propagators, we employ the operator $\bar{\psi} \gamma_{5} \gamma_{3} \psi$ for the $a_{1}$ and $\bar{\psi} \gamma_{3} \psi$ for the $\rho$. Wall sources and Coulomb gauge are also used. In figure 3 the Wilson valence masses for the $\rho$ and $a_{1}$ measured in simulation II, are plotted as a function of the valence hopping parameter $\kappa$. Linear fits are performed to both sets of data. The $\kappa_{c}$ is determined through the PCAC relation $m_{\pi}^{2}=C\left(1 / \kappa-1 / \kappa_{c}\right)$. We can see that the parity partners split less and less as $\kappa$ approaches $\kappa_{c}$. Similar behavior is also observed in partially quenched staggered hadron masses from this and earlier runs $\mid$.

In conclusion, the new $Q C D S P$ simulations have reproduced our previous runs successfully. The new simulation with $N_{f}=4, \beta=5.4$ and 


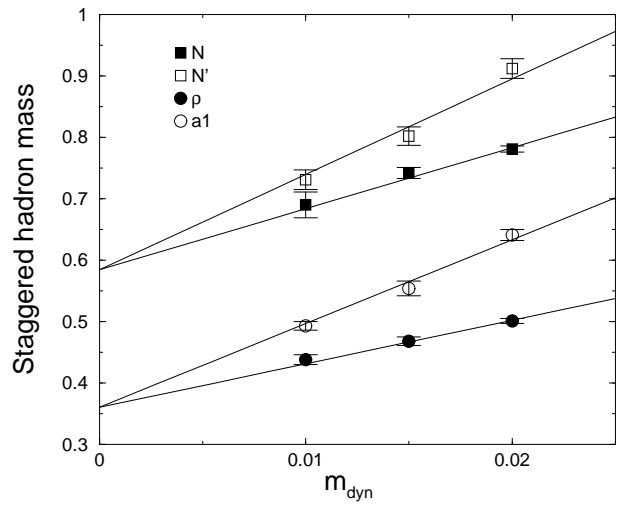

Figure 1. Staggered masses for the $\rho$ and $N$ and their parity partners vs. dynamical quark mass for $N_{f}=4$. The fits are three-parameter linear fits to six data points with common intercept.

$m=0.015$ on $Q C D S P$ produces results consistent with our previous potential discovery that chiral symmetry breaking is reduced substantially as $N_{f}$ increases from 2 to 4 . The Wilson valence hadron spectrum results are consistent with the hypothesis that this result is independent of lattice formalism, although more simulations are needed. The possible effects of small volume remain an important concern when interpreting our results. To resolve this issue we need to simulate on lattices of larger spatial volumes. In fact, larger, $24^{3} \times 32$, full QCD simulations are now under way.

\section{REFERENCES}

1. D. Chen, Nucl. Phys. B (Proc. Suppl.) 42 (1995), 312; Nucl. Phys. B (Proc. Suppl.) 47 (1996), 382. D. Chen and R. D. Mawhinney, Nucl. Phys. B (Proc. Suppl.) 53 (1997), 216.

2. R. D. Mawhinney, Nucl. Phys. B (Proc. Suppl.) 63 (1998), 212.

3. S. Gottlieb, et. al., Phys. Rev. D 38 (1988), 2245; M. Fukugita, et. al., Phys. Rev. D 47 (1993), 4739.

4. R. D. Mawhinney, hep-lat/9705031

5. S. Gottlieb, et. al., Phys. Rev. D 35 (1987), 2531.

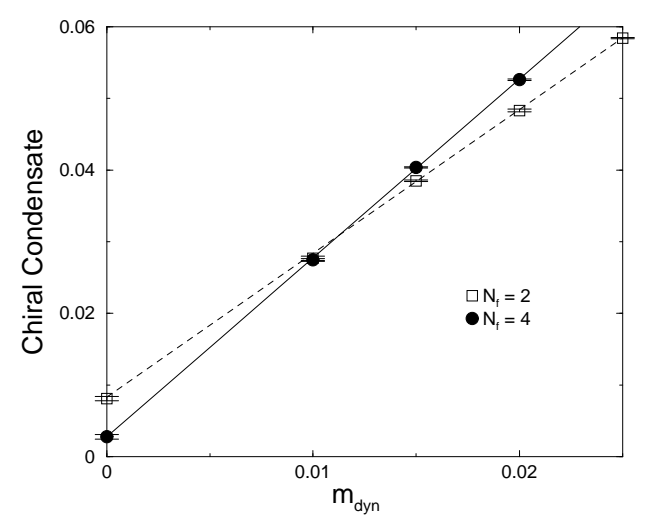

Figure 2. $\langle\bar{\psi} \psi\rangle$ vs. dynamical quark mass. Filled circles are for four flavors and open squares are for two flavors. The solid line is a linear fit to the 3 four flavor data points and the dashed line is a fit to the 4 two flavor data points.

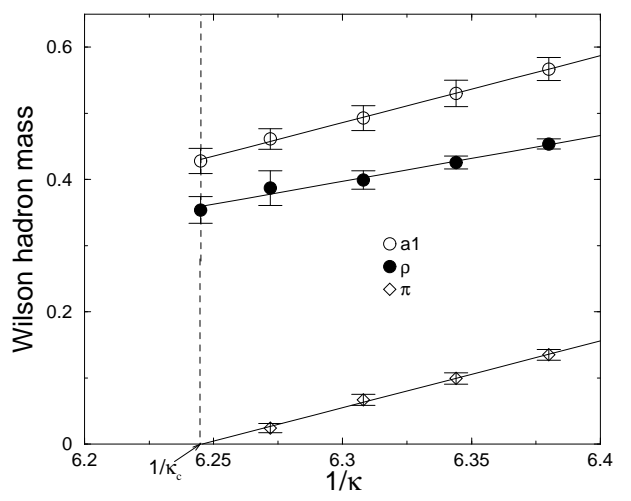

Figure 3. $m_{a_{1}}, m_{\rho}$ and $m_{\pi}^{2}$ vs. $1 / \kappa$ in run II with $N_{f}=4$ and $m_{\mathrm{dyn}}=0.015$. The filled circles are masses of $\rho$, open circles are masses of $a_{1}$ and diamonds are masses squared of $\pi$. The dashed vertical line shows the position of $\kappa_{c}$. 